\title{
Introduction to ChinaGrid Support Platform ${ }^{\star}$
}

\author{
Yongwei $\mathrm{Wu}^{1}$, Song $\mathrm{Wu}^{2}$, Huashan $\mathrm{Yu}^{3}$, and Chunming $\mathrm{Hu}^{4}$ \\ ${ }^{1}$ Department of Computer Science and Technology, \\ Tsinghua University, Beijing, 100084, China \\ ${ }^{2}$ Cluster and Grid Computing Lab, School of Computer, \\ Huazhong University of Science and Technology, Wuhan, 430074, China \\ ${ }^{3}$ School of Electronics Engineering and Computer Science, \\ Peking University, Beijing, 100871, China \\ ${ }^{4}$ School of Computer Science, \\ Beihang University, Beijing, 100083, China
}

\begin{abstract}
ChinaGrid aims at building a public service system for Chinese education and research. ChinaGrid Support Platform (CGSP) is a grid middleware developed for the construction of the ChinaGrid. Function modules of CGSP for system running are Domain Manager, Information Center, Job Manager, Data Manager, Service Container and Security Manager. Developing tools for gird constructor and application developers consist of Service Packaging Tool, Job Defining Tool, Portal Constructor and Programming API. CGSP architecture is introduced first. Then, CGSP function modules and developing tools are described. At last, job executing flow in CGSP is also put forward in the paper.
\end{abstract}

\section{Introduction}

As an important new research field, Grid [56] mainly focuses on resource sharing and coordinated problem solving in dynamic, multi-institutional virtual organization over the Network. In these several years, grid computing has been paid more and more attention all over the world. Many nation-wide grid projects have being constructed, such as TeraGrid 14, UK e-Science Program 12, NASA Information Power Grid (IPG) 13, K* Grid [16, ChinaGrid [12, CNGrid 15] and so on.

As the kernel of grid construction, grid middlewares are developed to shield heterogeneities and dynamic behaviors of all kinds of grid low-level resources. All grids described above put much more efforts on their grid middlewares. Corresponding achievement includes OGSI [20], WSRF [9], WSI [10] and Globus Toolkits (GT) series [711, Open Middleware Infrastructure Institute (OMII) [17], Tinghua Grid (TG) 18, CROWN [19].

ChinaGrid 112] aims at constructing public service system for Chinese education and research. ChinaGrid Support Platform (CGSP) 34 is a grid middleware

\footnotetext{
* This Work is supported by ChinaGrid project of Ministry of Education of China, Natural Science Foundation of China under Grant 60373004, 60373005, 90412006, 90412011, and National Key Basic Research Project of China under Grant 2004 CB318000.
} 
developed for building of the ChinaGrid. It integrates all sorts of heterogeneous resources, especially education and research resources distributed over China Education and Research Network (CERNET), to provide transparent and convenient grid services for science research and high education.

In moving towards this end, CGSP is developed based on following motivations:

- Provide a platform for grid construction from the top portal to the integration of bottom resources of grid. Not only does CGSP support the uniform management of heterogeneous resources, but it also supply the portal building, job defining, service packaging, and grid monitoring.

- Support secondary development of grid service and improve the flexibility of the system. Parallel programming interface and its running environment supply the complicated application development based on deployed services in grid.

- Follow latest grid standard and integrate existing advanced technology to avoid reduplicated works.

- Provide an extensible and reconfigurable grid framework, in order to fit the purpose of ChinaGrid to cover top 100 universities of China in the near future, and satisfy the autonomy of each ChinaGrid application or unit.

- Avoid unnecessary data delivery over grid. Data required by the computing job not stream with the job description file. It is delivered to the personal data space in data manager first and real computing nodes could get it directly when the job begins to be executed.

\section{CGSP Architecture}

CGSP is designed to provide grid toolkits for ChinaGrid application developers and specific grid constructors. It aims to reduce development cost of ChinaGrid as greatly as possible. It follows WSRF specification and is developed based on the core of GT3.9.1.

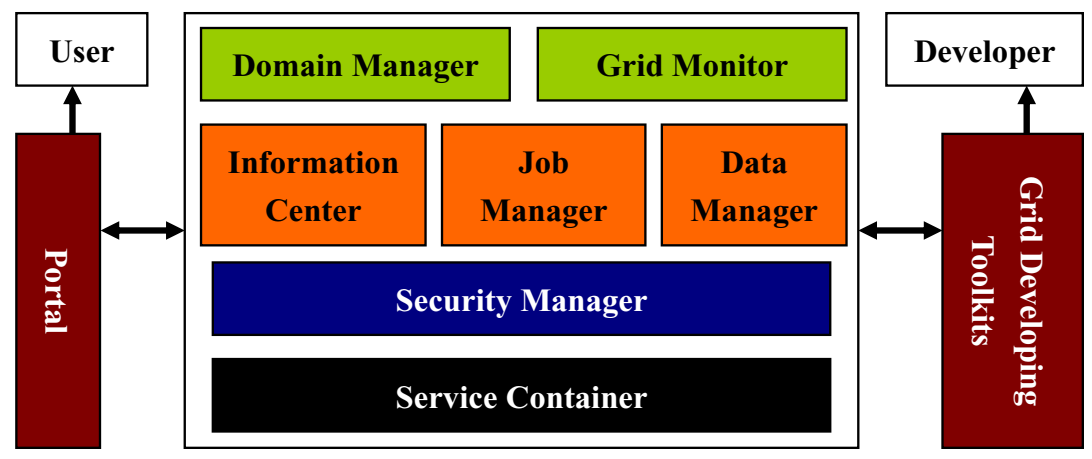

Fig. 1. CGSP Architecture 
In CGSP, domain is an independent grid, which could provide service for users by itself. Each domain has the same logical structure and consists of same CGSP function modules. ChinaGrid consists of multiple domains which are constructed into a tree structure based on CGSP. Fig. 1] shows the architecture of one domain in CGSP.

There are two types of users for CGSP. Normal end-users define, submit and monitor computing job through CGSP Portal. Others construct grid or develop complicated application by using high-level Grid Developing Toolkits.

Besides CGSP Portal and Grid Developing Toolkits, the kernel of CGSP can be divided into 4 layers shown as Fig. 1. Service Container produces a basic environment for the installation, deployment, running and monitoring of CGSP service in all kernel component nodes. Security manager focuses on the user identity authentication, identity mapping, service and resource authorization, and secure message passing between CGSP nodes. Information Center, Job Manager and Data Manager are composed to shield the heterogeneity of CGSP real computing nodes. Domain manager is in charge of the user management, log and accounting, and user identity mapping between different CGSP domains. Grid monitor mainly focuses on the monitoring of CGSP resources load, quality of services, user actions, job status and network, to ensure system running normally, and enhance grid perform as well.

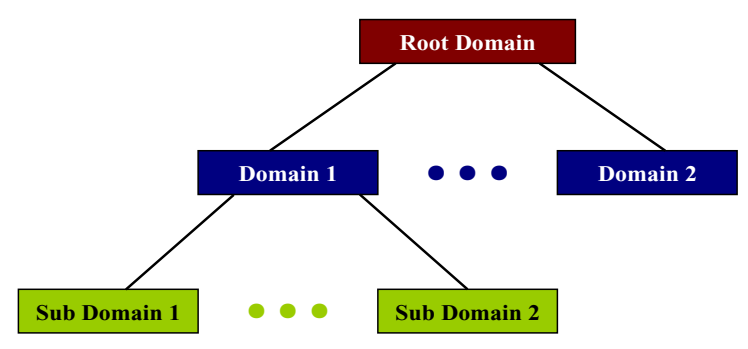

Fig. 2. CGSP Tree Structure

Based on CGSP, ChinaGrid could be constructed into a tree showed in Fig. 1. Each domain in it consists of the same CGSP components and could provide services for users by itself. Such a tree topology is maintained by the information center of CGSP totally. Each domain could get the global information through the tree query initiated by its information center and submit computing jobs to other domains directly under the help of the domain manager for the user identity mapping between different domains.

\section{CGSP Function Module}

There are 7 function modules in CGSP for system running. They are Domain Manager, Information Center, Job Manager, Data Manager, Service Container and Security Manager. 


\subsection{CGSP Portal}

The CGSP Portal is an extended Tomcat web server that can be run independently. In the ChinaGrid System, each domain contains one Portal and the grid applications should be deployed as a web component (Portlet, JSP/Servlet etc.) in the Portal and interacts with the end users with these components. Through portal, normal CGSP users could define, submit and monitor computing jobs easily, and manage personal data as well.

\subsection{Domain Manager}

Domain Manager is the management module of domains. Its functions include user database maintenance and processing request for user registration, authentication, user information query and modification. Cross-domain identity mapping, user behavior logging and accounting are also involved in this manager. Furthermore, CGSP component monitoring is used to make sure that each CGSP component (such as Job Manager, Information Center) are working normally.

\subsection{Grid Monitor (GM)}

ChinaGrid is a complex system and therefore, monitoring is essential for understanding their operation, debugging, failure detection, scheduling policy decision and for performance optimization. GM could provide information about the current state of various grid entities as well as to provide notification when certain events (e.g., system failures, performance problems, etc.) occur.

\subsection{Information Center (IC)}

IC provides service registration, publish, metadata management, service searching, service matching and resources status collection for the CGSP services in a uniform way. Each domain has its own IC. Multiple ICs in ChinaGrid are organized in a tree structure. Based on it, searching a service in multiple domains becomes available. It is also used for fault tolerance of service manager. IC plays a key role in service detection and the system status monitoring of the ChinaGrid. Its main functions include:

- Organizing and monitoring software and hardware resources

- Registering and publishing grid services

- Updating and monitoring service status

- Discovering and retrieving service

To balance its function and performance, IC is designed to store relatively static or long-standing information. When necessary, information could be collected and maintained via a "push/pull" way between Service Container and IC. 


\subsection{Job Manager (JM)}

JM focus on the job scheduling and execution management in CGSP. JM accepts users' requests in the form of SOAP message, and then interacts with IC to select and invoke relevant service according to "job description". Meanwhile, JM supports the work flow. JM is in charge of the process control of the work flow job, including parsing and compiling work flow description. The "rules" of the process of execution, including the process information, parallel distribution strategy and exception disposing rules, are described with the Grid Job Description Language (GJDL) customized based on BPEL by ourselves. In addition to controlling job's work flow, JM also monitors the status of jobs. The execution of the job in JM is completed through general running service deployed on the real computing nodes.

\subsection{Data Manager (DM)}

The main responsibilities of DM are to manage the storage resources and user data in the Grid and provide data service for users. In the view of system, DM is divided to three levels: data service access interface, metadata manager and storage resources. The function of it is to shield from users the heterogeneous underlying storage resources through the uniform data transfer protocol. And it provides a uniform storage resource access mode. The data service provided by metadata manager can shield the physical data storage path from users and organize the data space for them. So the users get a transparent data access. The targets of DM are as follows:

- Uniform data view based on user

- Uniform storage resource access mode

- Extendable storage architecture

- Data replica management

- High effectual and reliable data transfer mechanism

\subsection{Security Manager (SM)}

This module provides security functions, including identity authority, proxy certificate management, container and service level security and user access control. The Certificate Authority signs certificates for ChinaGrid entities to provide the basic security protection. Proxy management allows credential transferring among user, JM and CGSP services. Through implementing GSI Secure Conversation and GSI Secure Message, CGSP ensures the job submitted is legal and provides the security of job during job execution.

\subsection{Service Container (SC)}

$\mathrm{SC}$ is a basic runtime environment of the services and supply "service oriented facilities for computing", and it will be deployed on every node which supports the services. SC achieves core functions of the services including deployment, runtime, 
management, service status monitor. Besides acting as the basic Web Service Container, it also supports the life cycle management, asynchronous notification, remote deployment and hot deployment, batch job process etc.

The implementation of the SC is based on the core of Globus Toolkit 3.9.1, which includes the implementation of Web service core component because of the limited time and can not supply the necessary high level services for WSRF. Considering the requirement of CGSP, we extend and enhance the implementation of GT3.9.1 WSRF Core, and supply the necessary services properties for the running of ChinaGrid, such as remote and automatic deployment, hot deployment and general running service.

\section{CGSP Developing Tools}

The CGSP Grid Development Toolkits includes the CGSP Service Package Tool, Job Definition Tool, Portal Development Tool, Programming API (GridPPI) 21] and the corresponding running environment, visual administration/monitoring software, CGSP Installation Tool for remote service deployment and configuration. This package hides the underlying implementation of the CGSP's major functions and provides simple visual tool and programming interfaces, which makes the development of professional grid application easier. The major objectives of the software package for development are list as follows:

- Provide a set of visual resource packaging tool for developers to encapsulate the computing resources, software into CGSP services following ChinaGrid service specification

- Supply visual user interface to complete the design of work flow and generate XML job description files that satisfy with the GJDL and submitted to the job management for execution

- Provide a portal development framework

- Give out a programming specification and programming APIs which are used for grid user to develop grid applications with the support of services in grid system. The applications can run in the grid system

- Implement a graphics user interface for administrator to manage the grid system

- Provide a CGSP installation tool to ease and guide the installation of CGSP

\section{CGSP Job Executing Flow}

Fig. 3 shows the running flow of CGSP in a domain. Generally a grid application can be classified into three parts: resources (including computing resources, storage resources, software and legacy resources, data resources, etc.), jobs and interactions. First of all, the grid service containers should be deployed into the resources, and the resources will be extracted through service packaging tool, then these services should be deployed in the Service Containers (as depicted in figure 3, S1, S2, S3 represent respectively command-line style software, legacy program and service). 
For the special resource-storage, data manager is used to provide logical storage space for users.

Then, we need to use job defining tool for modeling a typical work flow into a job described by the GJDL, which in turn will be sent to the Job Manager. Because the job requests are via SOAP, the Job Manager is deployed over the Service Container which is responsible for the essential service processes. Job Manager is responsible for job request analysis, service selection, job scheduling and execution management, etc.

Thirdly, each domain needs to deploy Service Manager which collects service information from containers to support other software modules. Besides, each domain has to deploy Domain Manager to implement the functions of user management and other issues.

Lastly, one or more grid portals can be deployed to satisfy specific needs of different specialties. Portal developing tool can help users to develop web applications satisfy the requirement of interaction and can be deployed in the portal.

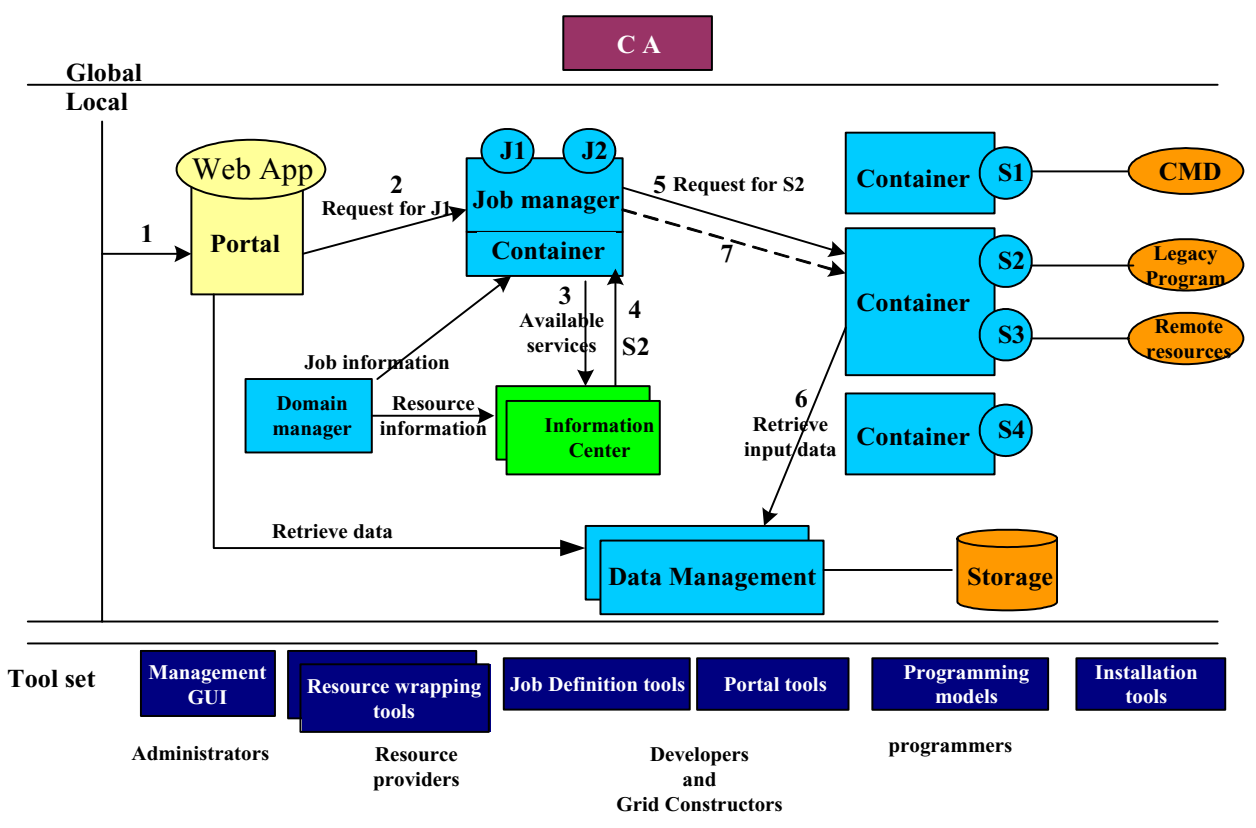

Fig. 3. CGSP Job Executing Flow

After an application is deployed, the steps of process are:

1. Firstly, a user should register and acquire the certificate signed by ChinaGrid. Then, the user logins the grid portal through a certain security protocol (such as HTTPS) and accomplishes the interaction with grid system through web applications deployed in the Portal. 
2. If necessary, user should upload the input data required by the computing job/request to the personal data space in data manager through Portal. Then, user could define a job (such as J1) by using job defining tool in Portal. At last the GJDL file generated by the Portal will be sent to the Job Manager.

3. According to the job process rule of J1 which is stored locally, the Job Manager queries available services from Information Center and contribute to the accomplishment of job $\mathrm{J} 1$.

4. Information Center collects the service information using the "Push/Pull" model. It maintains resource view in time, and so could return an available service list sorted by a certain rule. In principle, Information Center will return local available services. If necessary, it will query other Information Centers for more available services if it can not find any service satisfying the requirement in its own domain.

5. Job Manager selects service S2, and sends the service query to corresponding real computing node.

6. After Service Containers receive the requests, they need to invoke the access interfaces provided by the Data Manager in order to get the data by logical file path in the unified data space. Then the Service Containers execute the services.

7. Job Manager continuously collects the services status in the execution processes to report to the user through Portal in time.

8. At last, when the job is completed, the service container will put the computing results to personal data space in Data Manager.

\section{Conclusion}

CGSP provides a platform for ChinaGrid construction from the top portal to the bottom computing nodes. Not only does it support the uniform management of heterogeneous resources, but it also supplies the portal building, job defining and service packaging. Support secondary development of grid service and improve the flexibility of the system. In addition to supply the portal to ChinaGrid normal users, CGSP achieves parallel programming interface based on deployed services in CGSP for developing complicated applications.

CGSP satisfies the expansion, autonomy and localization of ChinaGrid. It consists of 7 function modules for system running and 4 application developing and programming tools.

This paper has given a high-level overview and snapshot summary of the current state of CGSP. Readers are warned that much detailed and important information has been omitted, and the new releases and previews represent significant changes. These considering using CGSP are therefore encouraged to obtain up-to-date and full information from the website www.chinagrid.edu.cn/CGSP.

\section{Acknowledgement}

We wish to express our sincere appreciation to the Prof. Hai Jin, Prof. Weimin Zheng, Prof. Xiaomin Li, Dr. Xiaowu Chen and other ChinaGrid experts for ex- 
tending their generous support for the successful conduct of the CGSP. Special thanks to CGSP working group members for providing materials for this paper.

\section{References}

1. ChinaGrid, http://www.chinagrid.edu.cn.

2. H. Jin, ChinaGrid: Making Grid Computing a Reality, Proceedings of ICADL 2004, Lecture Notes of Computer Science, (2004), 3334, 13-24

3. ChinaGrid Support Platform, http://www.chinagrid.edu.cn/CGSP.

4. CGSP Work Group, Design Specification of ChinaGrid Support Platform, Tsinghua University Press, Beijing, China, 2004

5. I. Foster, C. Kesselman, S. Tuecke, The Anatomy of the Grid: Enabling Scalable Virtual Organization, International J. Supercomputer Applications, 15(3), (2001)

6. I. Foster, C. Kesselman, The Physiology of the Grid: An Open Grid Services Architecture for Distributed Systems Integration, J. Nick, S. Tuecke, (2002)

7. I. Foster, C. Kesselman, Globus: A Metacomputing Infrastructure Toolkit, International J. Supercomputer Application, (1997), 11(2), 115-128

8. Open Grid Services Architecture (OGSA), https://forge.gridforum.org/projects/ ogsa-wg, or http://www.globus.org/ogsa/

9. Web Service Resource Framework (WSRF), http://www.globus.org/wsrf/ and http://www.ggf.org/documents/GFD.30.pdf

10. Web Services Interoperability (WSI), http://www.ws-i.org/

11. Globous Toolkits, http://www.globus.org.

12. UK e-Science Program, http://www.rcuk.ac.uk/escience/;

13. NASA Information Power Grid, http://www.ipg.nasa.gov/;

14. TeraGrid, http://www.teragrid.org/;

15. China National Grid, http://www.cngrid.cn;

16. $\mathrm{K}^{*}$ Grid, http://gridcenter.or.kr/

17. Open Middleware Infraxtructure Institute, http://www.omii.ac.uk/

18. Dazheng Huang, Fei Xie, Guangwen Yang, T.G.: a Market-oriented Computing System with Fine-grained Parallelism, 9th Workshop on Job Scheduling Strategies for Parallel Processing Seattle, Washington, (2002)

19. China Research and Development environment Over Wide-area Network, http://www.crown.org.cn

20. Tuecke, S., Czajkowski, K., Foster, I. , et.al.: Open Grid Services Infrastructure (OGSI) Version 1.0, Global Grid Forum Draft Recommendation. (2003).

21. Yongwei Wu, Guangwen Yang, Qing Wang, Weiming Zheng, Coarse-grained Distributed Parallel Programming Interface for Grid Computing, Lecture Notes in Computer Science, (2004), 3032, 255-258 